\title{
TEMA -2016: Prevalencia de la sarcopenia en Hospital de Día, Hospital Nacional de Geriatría y Gerontología, en el II semestre de 2012
}
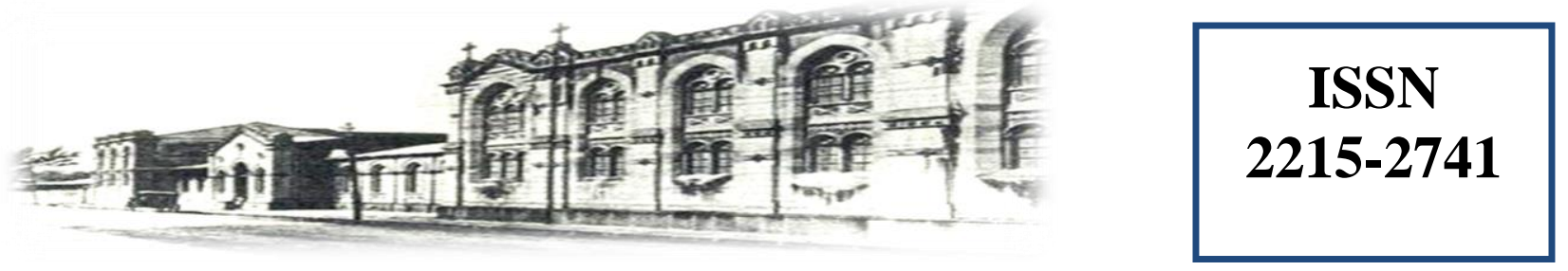

Hospital San Juan de Dios, San José, Costa Rica. Fundado en 1845

Recibido: $\quad 05 / 12 / 2016$

Aceptado: $\quad 09 / 12 / 2016$

Dionisio Flores Núñez ${ }^{1}$

${ }^{1}$ Médico Asistente Especialista en Geriatría y Gerontología, Hospital San Juan de Dios, Caja Costarricense del Seguro Social, correo electrónico: dionisiofn@gmail.com

\section{RESUMEN}

La sarcopenia es de alta prevalencia en la población adulta mayor y aumenta conforme aumenta la edad, generando complicaciones en los pacientes como aumento en el riesgo de caídas y fracturas, aumento en la morbimortalidad y deterioro funcional. En Costa Rica no se cuenta con estudios de investigación en sarcopenia. Este estudio determina la prevalencia de sarcopenia en pacientes adultos mayores del Servicio de Hospital de Dia del Hospital Nacional de Geriatría y Gerontología en el II semestre de 2012. La población estudiada fue mayor de 70 años y la mayoría de los que consultaron fueron mujeres. El estudio de la prevalencia de la sarcopenia documentó un tercio de los pacientes con este síndrome y que aumentaba conforme aumenta la edad. Más de la mitad de los pacientes sarcopénicos tienen alto riesgo de caídas, un estado cognitivo normal y sin datos clí- nicos de depresión. Y un porcentaje algo alarmante de dependencia de las actividades de la vida diaria.

\section{PALABRAS CLAVE}

Sarcopenia, deterioro funcional, adulto mayor, prevalencia.

\section{ABSTRACT}

Sarcopenia is a high prevalence in the adult population and increases as the age increases, causing complications in patients such as increased risk of falls and fractures, increased morbidity and mortality, and functional impairment. In Costa Rica there are no research studies on sarcopenia. This study determines the prevalence of sarcopenia in elderly of the Hospital de Día del Hospital 
Nacional de Geriatría y Gerontología in the second half of 2012. The population studied was older than 70 years and the majority of those consulted were women. The study of the prevalence of sarcopenia documented a third of patients with this syndrome and increased as the age increased. More than half of sarcopenic patients are at high risk for falls, a normal cognitive state and no clinical data on depression. And a somewhat alarming percentage of dependence on the activities of daily life.

\section{KEY WORDS}

Sarcopenia, functional impairment, elderly, prevalence.

\section{INTRODUCCIÓN}

Dado el crecimiento tanto a nivel nacional como mundial de la población adulta mayor el tema de sarcopenia es de vital importancia. Aunque la sarcopenia relacionada con la edad es frecuente y conlleva un costo personal y económico enorme, aún no cuenta con una definición clínica ampliamente aceptada, criterios diagnósticos de consenso o directrices terapéuticas.

Dada su importancia, es necesario conocer estadísticas nacionales acerca de su incidencia; no obstante, hasta hoy no se cuenta con su prevalencia y estudio poblacional. A nivel mundial, la población frágil será el doble que el actual para el año 2025. Como consecuencia del envejecimiento poblacional con un aumento de la longevidad, el número de personas mayores que se transformarán en sarcopénicos y frágiles requerirá de mayores instancias de cuidado a largo plazo y de institucionalización con mayor consumo de los recursos de salud. Por lo tanto al conocer la problemática institucional y/o nacional es importante generar políticas de prevención y manejo de los pacientes para mejorar su funcionalidad y calidad de vida con el apoyo interdisciplinario oportuno.

El estudio actual se desarrolla en el Servicio de Hospital de Día (HDD) del Hospital Nacional de Geriatría y Gerontología, San José, Costa Rica. Este es un servicio incorporado a los Servicios Integrales Geriátricos Ambulatorios (SIGA), por lo tanto dependiente de la Jefatura de Consulta Externa. Es un servicio geriátrico especializado de referencia, con una dinámica de trabajo interdisciplinaria: médicos geriatras, enfermería, trabajo social, terapia física, terapia ocupacional y terapia de lenguaje con eminente énfasis en rehabilitación. Sin embargo, también funciona como servicio de apoyo a la consulta externa, en los casos que requieran un esclarecimiento rápido del cuadro clínico o el ajuste de tratamientos crónicos. Una vez logradas las metas de rehabilitación se refieren los pacientes para continuar control ambulatorio en otros servicios.

El paciente ingresado al programa integral del HDD, se les realiza la historia geriátrica actualizada y se les aplican todas las pruebas geriátricas, psicológicas y de funcionalidad para obtener un marco global del paciente, con el fin de ofrecer metas de tratamiento médico y de rehabilitación funcional a corto plazo.

Una vez realizado este proceso, se debe de referir al paciente mediante interconsulta a terapia física, trabajo social y terapia ocupacional; enviar el laboratorio y gabinete que amerite si es el caso y dejar indicada la cita de seguimiento, idealmente en un plazo no mayor a tres semanas para revalorar el caso desde el punto de vista médico.

Este estudio de prevalencia de la sarcopenia fue enfocado en este servicio del Hospital, pues es el dedicado a la valoración geriátrica integral, tamizaje de pacientes frágiles, realización de pruebas clínicas, funcionales y psicológicas necesarias para documentar la presencia de la sarcopenia en una población vulnerable como lo son los pacientes ambulatorios que ingresan a este servicio como paciente con deterioro funcional agudo, subagudo o crónico.

\section{MATERIALES Y MÉTODOS}

El diseño del estudio es descriptivo, retrospectivo. Fue realizado en el Hospital de Día del Hospital Nacional de Geriatría y Gerontología en el II semestre del 2012. Los criterios de inclusión fueron los pacientes mayores de 60 años, hombres y mujeres, tener historia clínica geriátrica completa, test geriátricos, poseer la medición de la fuerza de prensión y velocidad de la marcha. 
En total los pacientes consultados fueron 866 expedientes, de los cuales588 fueron consultas totales como paciente nuevo de ingreso al programa de Hospital de Día. De esta población 286 pacientes fueron considerados dentro de los criterios de inclusión, 16 expedientes estaban incompletos por lo que el tamaño de la muestra se redujo a 270 pacientes.

Posterior al llenado de los formularios, los datos fueron ingresados en un formulario diseñado en "Epi Info 3.5" (CDC, 2010) para su posterior análisis. Dicho análisis se realizó en varias etapas. En la primera de ellas se realizó la descripción de las características generales de los pacientes evaluados con la determinación de frecuencias para obtener las distribuciones según grupo de edad y sexo. Seguidamente, se estimó la fuerza de presión según lateralidad y velocidad de la marcha, de acuerdo al grupo de edad y sexo de los pacientes evaluados para determinar la prevalencia de la sarcopenia. En la tabla 1 se describe la operacionalización de las variables.

En seguida, se evaluó la asociación entre la prevalencia de sarcopenia y, según las mediciones de independencia funcional, la presencia de diagnósticos de alto riesgo de mortalidad, riesgo de caídas, valoración cognitiva, riesgo de depresión, así como de las mediciones antropométricas por medio de la prueba de chi-cuadrado. Todos los análisis fueron desarrollados mediante "Stata 10.1" (Stata Corp., 2009 Texas, USA) y que se definió estadísticamente un valor de $\mathrm{p}$ de 0.05 .

\section{RESULTADOS}

En cuanto a la distribución general de la población estudiada se describe un total de 270 pacientes evaluados con la siguiente siguiente distribución etaria: $43.0 \%(116 / 270)$ fueron pacientes entre 70 y 79 años, seguido por el grupo de edad mayor o igual a 80 años que representa $40.4 \%$ (109/270) y, en menor porcentaje, las personas entre 60 a 69 años que significan $16.7 \%$ (45/270) de las personas En cuanto a la distribución general de la población estudiada se describe un total de 270 pacientes evaluados con la siguiente distribución etaria: $43.0 \%(116 / 270)$ igual a 80 años que representa 40.4\% (109/270) y, en menor porcentaje, las personas entre 60 a 69 años que significan $16.7 \%$ (45/270) de las personas en estudio. Por otro lado, la distribución según sexo evidenció ser más frecuentemente para el sexo femenino en el 79.6\% (215/270) sarcopenia fue evidente que la presencia aislada de la fuerza de prensión disminuida estuvo presente en $70.0 \%$ (189/270) de los casos y en 50.3\% (136/270) de ellos; la alteración en la velocidad de la marcha. La presencia de ambos criterios de sarcopenia estuvieron presentes en $33.7 \%$ (91/270) de los pacientes evaluados. En la tabla 2 se valora la distribución de pacientes adultos mayores estudidos por sospecha de criterios clínicos de sarcopenia.

En relación con la distribución de la presencia clínica de la sarcopenia según sexo, es preciso decir que en el grupo de los hombres se evidenció que la fuerza de prensión disminuida estuvo presente en $69.1 \%(38 / 55)$ y en $70.2 \%(151 / 215)$ en el grupo de las mujeres. En relación con la velocidad de la marcha, fue determinada en riesgo de sarcopenia $43.6 \%(24 / 55)$ en los hombres y $51.6 \%$ (111/215) dentro del grupo de las mujeres. Con respecto a la presencia de ambas, el resultado fue el siguiente: la alteración de la velocidad de la marcha y la fuerza de presión estuvo presente en $30.9 \%(17 / 55)$ de los hombres y en $34.4 \%$ (74/215) en el grupo de las mujeres.

Con respecto a la presencia de la fuerza de prensión disminuida según los grupos de edad, se evidenció que estuvo presente en $13 \%$ (36/270) de los casos entre 60 a 69 años, en $27.7 \%$ $(75 / 270)$ en el grupo entre 70 a 79 años y en $28.9 \%$ (78/270) de los casos con edades superiores o iguales a 80 años. La presencia de la velocidad de la marcha con riesgo de sarcopenia estuvo presente en 5.9\%(29/270) en el grupo de edad entre los 60 a los 69 años, en 17\% (70/270) en el grupo entre 70 a 79 años y en $26.3 \%$ (38/270) en el grupo de edad con 80 años o más.

Al evaluar la presencia de ambos criterios (presencia clínica de sarcopenia) según los tres grupos de edad, se evidenció que estuvo presente en $17.6 \%$ (16/91) en el grupo de 60 a 69 años, 38.5\% (35/91) en el grupo de 70 a 79 años y en $43.9 \%$ (40/91) en el grupo de edad más avanzada estudiado. 
Al evaluar la presencia de diagnósticos de alto riesgo de mortalidad a un año en todos pacientes evaluados, se describe que la condición más frecuente fue la diabetes en $27.4 \%$ (74/270), seguido de la depresión en $21.1 \%$ (57/270) y de la enfermedad cerebro vascular en $11.9 \%$ (32/270).

En relación con el número de comorbilidades relacionado con la presencia clínica de sarcopenia, se evidenció que la ausencia de diagnósticos de alto riesgo de mortalidad estuvo presente en $35.2 \%$ (32/91) de los casos, mientras que la presencia de comorbilidad fue de $37.4 \%$ (34/91). Finalmente, del grupo de dos diagnósticos y más en 27.5\% (25/91), la distribución evidenció contar con diferencias significativas entre los grupos $(\mathrm{p}<0.01)$.

En la presente sección se determina la asociación entre la presencia clínica de la sarcopenia y el estado funcional mediante la prueba de independencia funcional, en la que se describe que los pacientes sarcopénicos presentan 2.3\% (2/91) de asistencia moderada, $18.7 \%$ (17/91) asistencia mínima, $34.1 \%$ (31/91) necesidad de supervisión, 37.4\% (34/91) independencia modificada y 7.7\% (7/91) una independencia completa, lo que corresponde a un valor estadísticamente significativo.

En el estudio se describe que $92 \%$ de la población sarcopénica posee algún grado de dependencia física, comparado con 7,7\% de independencia completa a las actividades.

Ahora bien, los pacientes que no evidenciaron sarcopenia demuestran que la mayoría presenta independencia modificada cercana al 50\% de la muestra, mientras que un cuarto de los pacientes son independientes. Realizando una comparación entre los dos grupos, se describe claramente que los pacientes sarcopénicos poseen mayor dependencia funcional, comparada con los no sarcopénicos. Esta asociación ha sido descrita en la literatura internacional y es parte de las consecuencias de la presencia de este síndrome.

La asociación entre la presencia clínica de sarcopenia y el riesgo de caídas se describe un $52 \%$ (47/91) de la población estudiada con alto riesgo de caídas y 48\% (44/91) de ella con Tinetti mayor de 19 pts. (riesgo de caídas), con significancia estadística $\mathrm{p}<0.01$. Debido a estos datos, en este apartado se quiere valorar a los pacientes con sarcopenia y sin ella, ya que está bien descrita su asociación con caídas, por lo que es de importancia estadística para la investigación conocer la tendencia entre los dos grupos.

Por otro lado, los pacientes sin la presencia clínica de sarcopenia demostraron un bajo porcentaje de alto riesgo de caídas, a saber $15.6 \%$ (28/179); contrario a lo demostrado en el gráfico anterior de los pacientes sarcopénicos, de modo que queda demostrado que los pacientes sarcopénicos poseen mayor vulnerabilidad a caídas y que al final nadie está exento de ello; sin embargo, esto condiciona a mayores complicaciones a corto, mediano o largo plazo, ya que puede generarse un síndrome postcaída, mayor número de internamientos, aumento de la mortalidad o aumento del índice de fracturas.

En relación con la presencia clínica de sarcopenia y deterioro cognitivo se describe un $23.1 \%$ (21/91) presenta deterioro cognitivo moderado, mismo porcentaje descrito en deterioro cognitivo leve y un 53.8\% (49/91) una miniprueba del estado mental normal. Con un valor $\mathrm{p}$ de 0.09 estadísticamente no significativo.

Los pacientes con la presencia clínica de la sarcopenia asociada al riesgo de depresión, se ven relacionados en 41.8\% (38/91) con un Yesavage mayor de 5 y el 58.2\% (53/91) con un puntaje menor a 5 , lo que genera un valor $\mathrm{p}$ de 0.33

Esta asociación no es significativa, por lo que se necesitan más estudios de ella en otras poblaciones y escenarios.

Así pues, en la comparación de los componentes de sarcopenia según las categorías de IMC, se evidenció que ninguno de los componentes de forma individual, ni en conjunto, demostraron diferencias significativas entre los pacientes evaluados ( $p$ 0.52); sin embargo, la presencia de sarcopenia se presentó con mayor frecuencia en pacientes con un IMC normal, lo que refleja $34.1 \%$ (31/91) de la población en estudio, seguido del paciente sarcopénico en obesidad en $28.6 \%$ (26/91), $25.3 \%$ (23/91) con antropometría de sobrepeso y finalmente $12.1 \%$ (11/91) con características de bajo peso. 


\section{DISUCSIÓN}

En este sentido, se evidencia cómo, generalmente, son las mujeres quienes consultan más a los servicios médicos, pues este género suele ser más precavido y afanado en el cuidado de la salud. Durante los últimos 15 años se ha atendido alrededor de 1,4 mujeres por cada varón de 65 años y más. Esta población ejerce una presión importante sobre los servicios de consulta externa de la Caja Costarricense de Seguro Social (CCSS). De hecho, el número de consultas por habitante que recibe esta población solo es superado por el grupo de menores de un año, esto según reporte de "La salud de las personas adultas mayores en Costa Rica" en el año 2004, de la Organización Panamericana de la Salud.

La edad de los adultos mayores que consultan a los servicios de apoyo del Hospital Nacional de Geriatría, en especial en el servicio de Hospital de Día, representa un número mayoritario de personas mayores de 70 años, esto debido a que las edades entre 60-69 años con enfermedades crónicas están siendo vistas en su mayoría por especialistas de otras disciplinas diferentes a geriatría, tal vez porque tienen controles con esas especialidades desde años atrás y no hacen el cambio de atención en el hospital geriátrico. La variabilidad en la disminución de la fuerza de prensión en comparación con la velocidad de la marcha lenta, en donde la primera obtiene un porcentaje mayor, probablemente sea por la técnica usada y el expertis del terapeuta ocupacional que realiza la prueba en hospital de día, y la velocidad de la marcha realizada por varios exploradores con técnicas diferentes que podrían tener diferencias a la hora de aplicar las pruebas.

Tomando en consideración la presencia concomitante de las dos variables estudiadas (fuerza de prensión disminuida y velocidad de la marcha lenta) la prevalencia de la sospecha clínica de sarcopenia obtuvo porcentajes por encima del $30 \%$ (33.7\%), 34,4\% en mujeres y $30,9 \%$ en hombres. Un estudio de prevalencia de la sarcopenia publicado en 2002 y que evaluaba ambos sexos en la comunidad, documentó una prevalencia de $22,6 \%$ en mujeres y un $26,8 \%$ en hombres.

En relación con ese estudio, en la presente investigación se encontró que conforme aumenta la edad, mayor prevalencia de sospecha clínica de sarcopenia. El grupo de 60-69 años comparte un porcentaje similar al estudio anterior, y para el grupo de más de 80 años se reporta un porcentaje de $43.9 \%$ semejante al estudio planteado. Otro estudio demuestra que en la población muy envejecida de Nuevo México se reporta una relación de uno por cada dos pacientes con pruebas alteradas.

La comparación de resultados de los estudios no es posible por técnicas de medición diferentes, para uno la medición de la masa muscular apendicular y para el presente mediciones clínicas de aproximación diagnóstica. Sin embargo, son congruentes en que la edad es un factor importante en la prevalencia de la sarcopenia, ya que como se ha mencionado, este síndrome aumenta con la edad. No obstante, si se correlacionan los resultados con estudios clínicos de prevalencia de la sarcopenia a nivel internacional, se puede inferir que tanto Baumgartner et al (1998) como Melton et al (2000) y Morley et al (2001), este estudio comparte con el de dichos autores una prevalencia global similar.

Este estudio presenta una relación de acuerdo a la carga de comorbilidad con el riesgo de mortalidad a un año en los pacientes con sospecha de sarcopenia. Por ejemplo: quienes no tenían diagnóstico representan un $35.2 \%$ de los pacientes, con mortalidad de $9.5 \%$ a un año, diagnóstico correspondiente a $37.4 \%$ con riesgo de mortalidad a un año de $28.6 \%$. Este último representa una carga importante de pacientes en donde solo este diagnóstico, asociado a la presencia de sarcopenia, le confiere un alto porcentaje de mortalidad, tal y como se describe en la literatura y parte de las consecuencias de este síndrome.

En relación con el estado funcional medido por MIF al tomar en cuenta los dos criterios (presencia clínica de la sarcopenia), la mayoría (92.5\%) de los pacientes sarcopénicos presentan algún grado de limitación funcional. a saber, desde una independencia modificada como el bastón, andadera entre otros aditamentos para uso en actividades de la vida diaria, hasta requerir una asistencia mínima-moderada, lo que estadísticamente representa una suma significativa $(<0.01)$ entre la asociación de la presencia clínica de la sarcopenia y la limitación funcional que se traduce en discapa- 
cidad. Cabe mencionar que los pacientes que ingresan al Hospital de Día deben tener la capacidad de atención ambulatoria para el manejo de rehabilitación funcional y, es por eso, que casi no se presentaron pacientes con MIF menor a 72, lo que según los resultados revelan el perfil de paciente que asiste a este centro asistencial.

Por consiguiente, el presente estudio documenta una asociación clara entre los criterios clínicos de sarcopenia y el alto riesgo de caídas con significancia estadística $(<0.01)$. Con una prevalencia del $52 \%$ de alto riesgo de caerse y $48 \%$ de riesgo de caídas, lo que determina que los pacientes sarcopénicos tienen un riesgo de caerse, en comparación con los que no tenían sospecha clínica de sarcopenia, ya que en este grupo se reduce el riesgo. Igual al estudio japonés, se determinó la misma asociación entre las variables. Además, se debe tomar en cuenta que la población de HDD estudiada presenta cierto deterioro funcional y que en muchos casos el síndrome de caídas es el principal motivo de ingreso al programa de rehabilitación funcional integral.

Para efectos del presente estudio, casi la mitad de la población sarcopénica $(41.8 \%)$ se reportó con síntomas depresivos; sin embargo, entre la presencia clínica de sarcopenia y los síntomas depresivos no se documentó asociación de las variables con significancia estadística ( $p$ 0.33) de manera que los síntomas depresivos pueden ser reflejo de otras condiciones que afecten y atañen al adulto mayor, como pueden ser etiologías de carácter social, funcional o de comorbilidad, las cuales son comunes en esta población tan vulnerable.

Por lo que respecta al deterioro cognitivo, de un estudio de 2013 que evaluaba la sarcopenia asociada con el deterioro cognitivo en una comunidad de mujeres adultas mayores, resultó que $492 \mathrm{mu}$ jeres tenían deterioro cognitivo, $16,3 \%$, por lo que se concluyó que no existe evidencia de asociación significativa entre la sarcopenia y el deterioro cognitivo (Abellan van Kan G., Cesari M., GilletGuyonnet S. et al, 2013). Del mismo modo, se puede observar que en el estudio actual, el deterioro cognitivo tanto leve como moderado se documentó $46.2 \%$ en los pacientes sarcopénicos y sin encontrar significancia estadística $(>0.05)$ entre la asociación de presencia clínica de sarcope- nia y el deterioro cognitivo. Más bien, ese deterioro puede verse influenciado por múltiples condiciones que no son objetivo de este estudio y, simplemente, ser el resultado de información estadística que no se puede correlacionar entre sí como en los estudios internacionales.

Finalmente, un tercio de los pacientes sarcopénicos tienen un peso normal o adecuado que al asociarlos con disminución de la fuerza de prensión y velocidad de la marcha lo acerca a un número importante de pacientes en probable relación con obesidad sarcopénica, mas no es objetivo de la presente investigación la estimación de este concepto. Se necesitan más estudios para determinar su prevalencia mediante la utilización de una técnica de medición de la masa muscular conocida y ampliamente utilizada a nivel mundial, que en el medio donde se desarrolla la investigación es limitado.

\section{CONCLUSIONES}

La sarcopenia es de alta prevalencia en nuestra población y conlleva costes económicos enormes. Se debe trabajar en equipo multidisciplinario para mejorar en los pacientes el rendimiento físico, fuerza muscular y masa muscular y de esta forma generar un impacto en mejorar la calidad de vida, disminución de caídas y reducción de posibles hospitalizaciones e ingreso a residencias.

\section{BIBLIOGRAFÍA}

1. Abellan van Kan G, Rolland Y, Andrieu S, Bauer J, Beauchet O, Bonnefoy M, et al. Gait speed at usual pace as a predictor of adverse outcomes in community-dwelling older people: an International Academy on Nutrition and Aging (IANA) Task Force. J Nutr Health Aging 2009;13(10):881-889.

2. Cederholm, T., Bauer, J., Boirie Y., Schneider, S., et al., Toward a Definition of Sarcopenia. Clinics in Geriatric Medicine 27 (2011) 341-353.

3. Cruz, A., Baeyens, J., Bauer, J., et al. Sarcopenia: consenso europeo sobre su definición y diagnóstico. Informe del Grupo Europeo de trabajo sobre la sarcopenia en personas de edad avanzada. Age and ageing 39 (2010) 412-423. 
4. Janssen, Ian. The Epidemiology of Sarcopenia. Clinics in Geriatric Medicine 27 (2011) 355-363.

5. Desai, M, Bogardus, S., Williams, C., Vitagliano G., Inouye, G. Development and Validation of a Risk-Adjustment Index for Older Patients: The High-Risk Diagnoses for the Elderly Scale. Journal of American Geriatric Society 50:474-481, 2002.

6. Pillard, F., Laoudj-Chenivesse, D., Carnac G., Mercier, J., et al. Physical Activity and Sarcopenia. Clinics in Geriatric Medicine 27 (2011) 449-470.

7. Rolland, Y., Onder, G., Morley, J., GuilletGuyonet, S., et al. Current and Future Pharmacologic Treatment of Sarcopenia. Clinics in Geriatric Medicine 27 (2011) 423-447.

8. Rosenberg, Irwin H., Sarcopenia: Origins and Clinical Relevance. Clinics in Geriatric Medicine 27 (2011) 337-339.

9. Thomas, David R. Sarcopenia. Clinics in Geriatric Medicine 27 (2011) 331-346.

10. Tinetti ME, Williams TF, Mayewski R, Fall Risk Index for elderly patients based on number of chronic disabilities. Am J Med 1986:80:429-434.

11. Visser, M., Schaap, L. Consequences of Sarcopenia. Clinics in Geriatric Medicine 27 (2011) 387-399.

12. Waters, Debra. L., Baumgartner Richard N. Sarcopenia and Obesity. Clinics in Geriatric Medicine 27 (2011) 401-421.

13. Walrand, S., Guillet, C., Salles, J., Cano, N., et al. Physiopathological Mechanism of Sarcopenia. Clinics in Geriatric Medicine 27 (2011) 365-385.

14. Rosenberg I. Summary comments: epidemiological and methodological problems in determining nutritional status of older persons. Am J Clin Nutr 1989; 50: 1231-3.

15. Rosenberg IH. Sarcopenia: origins and clinical relevance. J Nutr 1997; 127: 990S-91S.

16. Morley JE, Baumgartner RN, Roubenoff R et al. Sarcopenia. J Lab Clin Med 2001; 137: 231-43.

17. Inouye SK, Studenski S, Tinetti ME et al. Geriatric syndromes: clinical, research, and policy implications of a core geriatric concept. J Am Geriatr Soc 2007; 55: 780-91.

18. Olde Rikkert MG, Rigaud AS, van Hoeyweghen RJ et al. Geriatric syndromes: medical misnomer or progress in geriatrics? Neth $\mathbf{J}$ Med 2003; 61: 83-7.

19. Cruz-Jentoft A, Landi F, Topinková E et al. Understanding sarcopenia as a geriatric syndrome. Curr Opin Clin Nutr Metab Care 2010; 13: 1-7.

20. Iannuzzi-Sucich M, Prestwood KM, Kenny AM. Prevalence of sarcopenia and predictors of skeletal muscle mass in healthy, older men and women. J Gerontol A Biol Sci Med Sci 2002; 57: M772-7.

21. Chien MY, Huang TY, Wu YT. Prevalence of sarcopenia estimated using a bioelectrical impedance analysis prediction equation in community-dwelling elderly people in Taiwan. J Am Geriatr Soc 2008; 56: 1710-5.

22. Tanimoto Y, Watanabe M, Sun W. et al. Sarcopenia and falls in community-dwelling elderly subjects in Japan: Defining sarcopenia according to criteria of the European Working Group on Sarcopenia in Older People. Arch Gerontol Geriatr. 2014 May 4. pii: S0167-4943(14)00062-4

23. Vetrano DL, Landi F, Volpato S, et al. Association of Sarcopenia With Short- and Longterm Mortality in Older Adults Admitted to Acute Care Wards: Results From the CRIME Study. J Gerontol A Biol Sci Med Sci. 2014 Apr 17

24. Alley DE, Shardell MD, Peters KW, et al. Grip strength cutpoints for the identification of clinically relevant weakness. J Gerontol A Biol Sci Med Sci. 2014 May;69(5):559-66.

25. Hsu YH, Liang CK, Chou MY, et al. Association of cognitive impairment, depressive symptoms and sarcopenia among healthy older men in the veterans retirement community in southern Taiwan: a cross-sectional study. Geriatr Gerontol Int. 2014 Feb;14 Suppl 1:102-8.

\section{CONFLICTO DE INTERÉS Y/O AGRADE- CIMIENTOS}

Los autores declaran que no existió ningún conflicto de interés en el presente reporte. 


\section{ANEXOS}

Tabla 1. Operacionalización de las variables

\begin{tabular}{|c|c|c|c|c|}
\hline & OBJETIVO & VARIABLE & $\begin{array}{l}\text { INSTRUMENTO O } \\
\text { CARACTERISTICA } \\
\text { PARA MEDICIÓN. }\end{array}$ & VALORES UTILIZADOS \\
\hline \multirow[t]{2}{*}{1.} & \multirow{2}{*}{$\begin{array}{l}\text { Describir el } \\
\text { grupo de } \\
\text { adultos } \\
\text { mayores } \\
\text { estudiados, por } \\
\text { edad y sexo } \\
\end{array}$} & Sexo & Sexo & $\begin{array}{l}\text { Femenino } \\
\text { Masculino }\end{array}$ \\
\hline & & Edad & Años cumplidos & $\begin{array}{l}60-69 \text { años } \\
70-79 \text { años } \\
>80 \text { años }\end{array}$ \\
\hline 2. & $\begin{array}{l}\text { Estimar la } \\
\text { prevalencia de } \\
\text { la sospecha } \\
\text { clínica de la } \\
\text { sarcopenia, } \\
\text { según sexo y } \\
\text { grupo etáreo. }\end{array}$ & $\begin{array}{l}\text { Prevalencia } \\
\text { indicadores } \\
\text { clínicos, } \\
\text { porcentaje, } \\
\text { según sexo y } \\
\text { edad } \\
\end{array}$ & $\begin{array}{l}\text { Velocidad marcha } \\
\text { Fuerza de prensión }\end{array}$ & $\begin{array}{l}<0,6 \mathrm{~m} / \mathrm{seg} \\
<30 \mathrm{Kg} \text { hombres } \\
<20 \mathrm{Kg} \text { mujeres }\end{array}$ \\
\hline & \multirow{6}{*}{$\begin{array}{l}\text { Factores } \\
\text { asociados a la } \\
\text { sospecha } \\
\text { clínica de la } \\
\text { sarcopenia. }\end{array}$} & $\begin{array}{c}\text { Diagnósticos } \\
\text { de alto riesgo } \\
\text { mortalidad }\end{array}$ & $\begin{array}{c}\text { Escala diagnósticos } \\
\text { de alto riesgo de } \\
\text { mortalidad en AM }\end{array}$ & $\begin{array}{crcr}\text { Diagnósticos / } & \text { Mortalidad a } 1 \text { año } \\
0 & 9,5 \% & 6 & 69,2 \% \\
1 & 28,6 \% & 7 & 72,7 \% \\
2 & 32,5 \% & 8 & 66,7 \% \\
3 & 40,7 \% & 9 & 75 \% \\
4 & 55 \% & 10 & 100 \% \\
5 & 55,6 \% & & \\
\end{array}$ \\
\hline & & $\begin{array}{l}\text { Estado } \\
\text { funcional } \\
\text { físico }\end{array}$ & $\begin{array}{l}\text { Puntaje medida de } \\
\text { independencia } \\
\text { funcional (MIF) }\end{array}$ & $\begin{array}{l}18-30 \text { pts. asistencia total } \\
36-45 \text { pts. asistencia máxima } \\
54-63 \text { pts. asistencia moderada } \\
72-80 \text { pts. asistencia mínima } \\
90-100 \text { pts. necesidad de supervisión } \\
108-120 \text { pts. independencia modificada } \\
126 \text { pts. independencia completa }\end{array}$ \\
\hline & & $\begin{array}{l}\text { Riesgo de } \\
\text { caidas }\end{array}$ & Puntaje Tinetti & $\begin{array}{l}\text { Menor } 19 \text { alto riesgo de caídas } \\
\text { Mayor } 19 \text { riesgo de caídas }\end{array}$ \\
\hline & & $\begin{array}{l}\text { Deterioro } \\
\text { cognitivo }\end{array}$ & $\begin{array}{l}\text { Puntaje Mini Mental } \\
\text { Test (MMSE) }\end{array}$ & $\begin{array}{l}24 \text { a } 30 \text { normal } \\
21 \text { a } 23 \text { déficit leve } \\
11 \text { a } 20 \text { déficit moderado } \\
0 \text { a } 10 \text { déficit severo }\end{array}$ \\
\hline & & $\begin{array}{l}\text { Riesgo de } \\
\text { depresión }\end{array}$ & $\begin{array}{c}\text { Puntaje escala } \\
\text { depresión geriátrica } \\
\text { (Yesavage) }\end{array}$ & $\begin{array}{l}0-4 \text { puntos normal } \\
>5 \text { sospecha de depresión }\end{array}$ \\
\hline & & $\begin{array}{l}\text { Estado } \\
\text { nutricional }\end{array}$ & $\begin{array}{l}\text { Índice de masa } \\
\text { corporal }\end{array}$ & $\begin{array}{l}<23 \text { bajo peso } \\
23,1 \text { a } 27,9 \text { normal } \\
28-31,9 \text { sobrepeso } \\
>32 \text { obesidad }\end{array}$ \\
\hline
\end{tabular}

Fuente: Elaboración propia 
Tabla 2. Distribución de pacientes adultos mayores estudiados por sospecha de criterior clínicos de sarcopenia. Hospital de Día, Hospital Nacional de Geriatría y Gerontología, II semestre 2012.

\begin{tabular}{lcc} 
Criterios de sarcopenla & N & \% \\
\hline $\begin{array}{lcc}\text { Fuerza de presión } \\
\text { Disminuido }\end{array}$ & 189 & 70.0 \\
Normal & 81 & 30.0 \\
\hline Total & $\mathbf{2 7 0}$ & 100 \\
Velocidad de la marcha & & \\
$<0,6 \mathrm{~m} / \mathrm{seg}$ & 136 & 50.3 \\
$>0,6 \mathrm{~m} / \mathrm{seg}$ & 134 & 49.6 \\
\hline Total & $\mathbf{2 7 0}$ & $\mathbf{1 0 0}$ \\
\hline Presencia de 2 criterios & 91 & 33.7 \\
\hline
\end{tabular}

Fuente: Expedientes clínicos HNGG 
